\title{
Solution of Dirichlet Boundary Value Problem by Mellin Transform.
}

\author{
George .N. Emenogu \\ Department of mathematics Michael Okpara University of Agriculture Umudike
}

Abstract: An infinite slab subject to temperature variation is analyzed, the problem is formulated using conformal mapping and solved by mellin transform and method of residue. A closed form solution for the temperature distribution is obtained .A detailed verification of the solution is carried out and find to satisfying the Laplace equation.

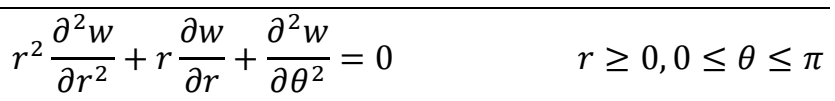

\section{Introduction}

To illustrate the use of the Mellin transform in the solution of a physical problem, L.N. Sneddon [1] showed how the distribution of potential in an infinite wedge without a crack can be computed. Analysis of an elastic wedge under out of plane stress is carried out by G.N Emenogu and J.N Nnadi.[2] .In this paper, we investigated an infinite slab A,B,C,D,E,F of thickness a, subjected to temperature $\emptyset(x, a)=T$ along BC and to $\varnothing(x, 0)=2 T$ along $\mathrm{DE}, \emptyset(x, a)=0$ along $\mathrm{AB}$ and $\emptyset(x, 0)=0$ along $\mathrm{EF}$.the problem is to find the temperature distribution within the entire slap

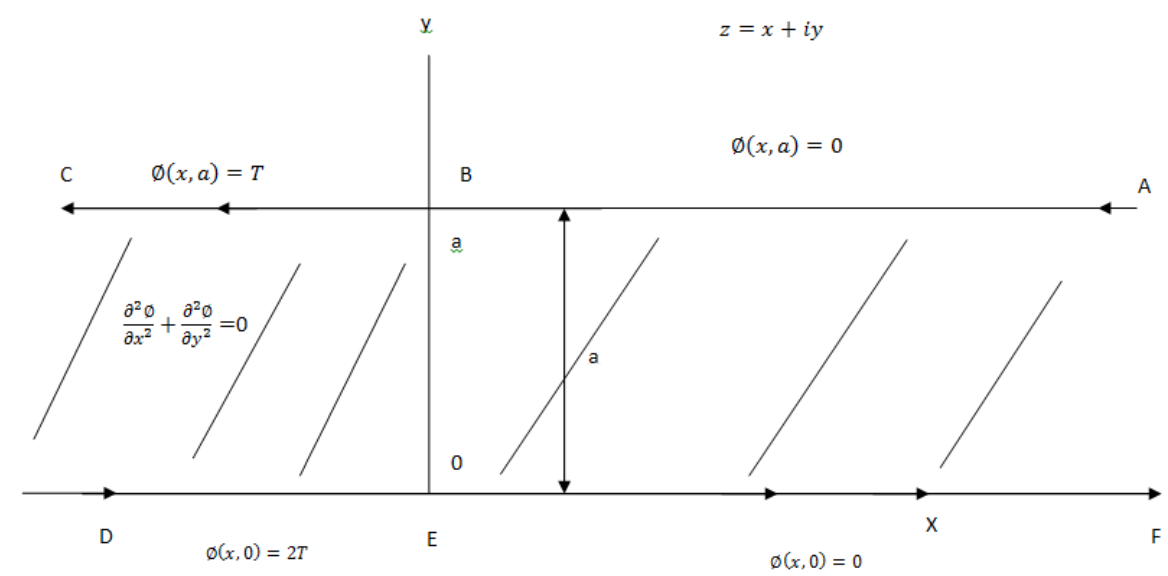

Fig1 Geometry of the plane that forms the slab

The problem in mathematical terms is that of finding a formula for the temperature distribution $\emptyset(x, y)$ so that the following boundary value problem for the Laplace equation is solved

$$
\begin{array}{rlc} 
& \frac{\partial^{2} \emptyset}{\partial x^{2}}+\frac{\partial^{2} \emptyset}{\partial y^{2}}=0, & -\infty<x<\infty, 0 \leq y \leq a \\
=0, & \varnothing(x, a)=T, \quad x>0 & -\infty<x \leq 0 \\
=0, & \varnothing(x, 0)=2 T, \quad x>0 & -\infty<x \leq 0
\end{array}
$$

\section{A transformation of the plane representing the slab}

Because of the nature of the boundary conditions, elementary methods of solution such as method of separation of variables cannot be applied. Therefore, the region of analysis is transformed to a region where integral transform can be applied 


\subsection{The Conformal Transformation}

$$
w(z)=e^{\frac{\pi z}{a}}, \quad z=x+i y
$$

Maps the shaded plane region in fig 1 onto the upper half w-plane of Fig 2

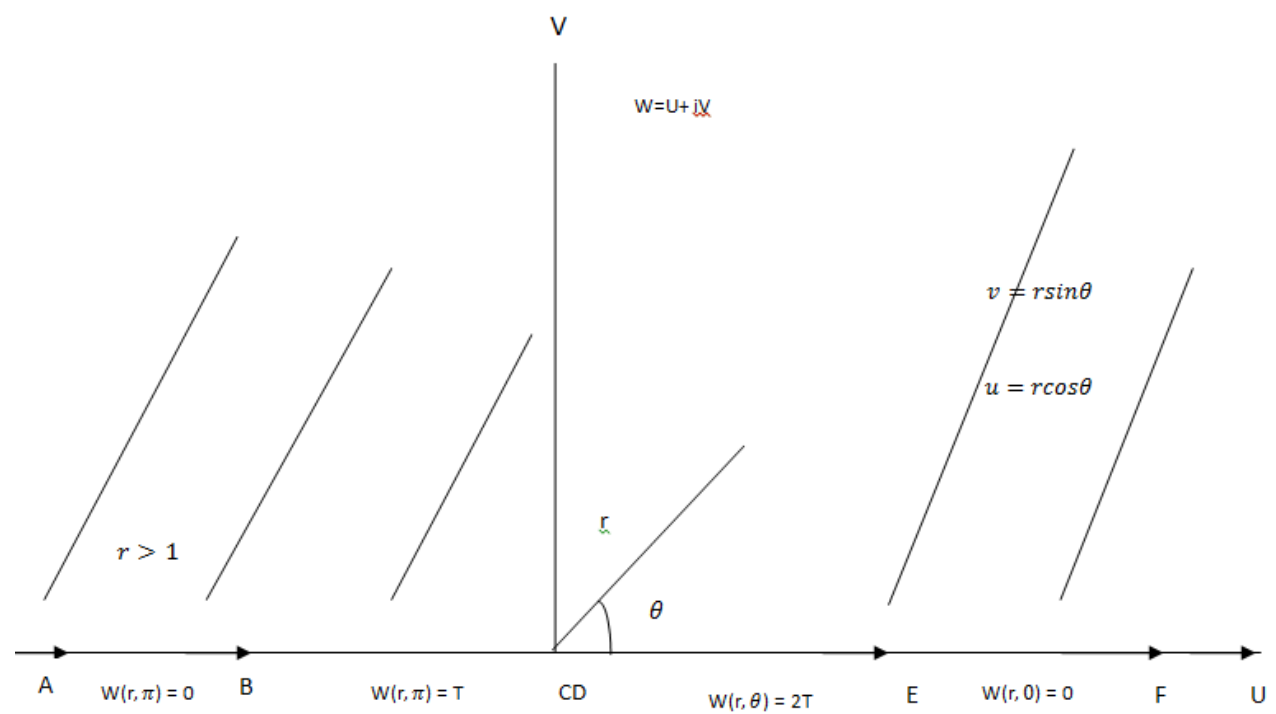

\section{2 transformation of the problem}

The conformal mapping which transforms the infinite slab into the upper half plane also changes the boundary value problem (1.1), (1.2) and (1.3) such that.

That is

$$
w(u, v)=\emptyset(x, y)
$$

$$
w(u(x, y), v(x, y))=\emptyset(x, y)
$$

Where

$$
\begin{array}{ll}
-\infty \leq u(x, y) \leq \infty, & v(x, y) \geq 0 \\
-\infty \leq x \leq \infty, & 0 \leq y \leq a
\end{array}
$$

Following 2.3, the boundary conditions in terms of $\mathrm{w}$ becomes

$$
\left.\begin{array}{c}
w(u(x, a), v(x, a))=\varnothing(x, a)=T \\
w(u(x, 0), v(x, 0))=\varnothing(x, 0)=2 T
\end{array}\right\}
$$

The function $\mathrm{w}$ is written as a complex variable in the form

$\mathrm{W}$ is also written in polar form as

$$
w(u, v)=u+i v
$$

$$
\begin{gathered}
w(r, \theta)=r e^{i \theta}, \text { where } \\
u(r, \theta)=r \cos \theta \text { and } v(r, \theta)=r \sin \theta
\end{gathered}
$$

To link $u$ and $v$ to $w(x, y)$, we note that

Hence

$$
\begin{aligned}
w(x, y) & =e^{\frac{\pi}{a}(x+i y)} \\
& =e^{\left(\frac{\pi}{a} x+i \frac{\pi}{a} y\right)} \\
& =e^{\frac{\pi}{a} x} \cdot e^{i \frac{\pi}{a} y} \\
= & e^{\frac{\pi}{a} x}\left(\cos \frac{\pi}{a} y+i \sin \frac{\pi}{a} y\right) \\
= & e^{\frac{\pi}{a} x \cos \frac{\pi}{a} y}+i e^{\frac{\pi}{a} x \sin \frac{\pi}{a} y} \\
= & u+i v
\end{aligned}
$$

$$
\begin{gathered}
u(x, y)=e^{\frac{\pi}{a} x} \cos \frac{\pi}{a} y \text { And } v(x, y)=e^{\frac{\pi}{a} x} \sin \frac{\pi}{a} y \\
u(x, 0)=e^{\frac{\pi}{a} x} \cos 0 \quad \text { And } v(x, 0)=0 \\
u(x, 0)=e^{\frac{\pi}{a} x} \quad \text { and } v(x, 0)=0
\end{gathered}
$$

On the other hand

$$
w(r, \theta)=r \cos \theta+i \sin \theta
$$

Implies

$$
u(x, y)=e^{\frac{\pi}{a} x} \cos \frac{\pi}{a} y=r \cos \theta
$$


Hence

$$
v(x, y)=e^{\frac{\pi}{a} x} \sin \frac{\pi}{a} y=r \sin \theta,
$$

Then

$$
\begin{aligned}
& \left(e^{\frac{\pi}{a} x} \cos \frac{\pi}{a} y\right)^{2}=r^{2} \cos ^{2} \theta \\
& \left(e^{\frac{\pi}{a} x} \sin \frac{\pi}{a} y\right)^{2}=r^{2} \sin ^{2} \theta
\end{aligned}
$$

Or

$$
e^{2 \frac{\pi}{a} x} \cos ^{2} \frac{\pi}{a} y+e^{2 \frac{\pi}{a} x} \sin ^{2} \frac{\pi}{a} y=r^{2} \cos ^{2} \theta+r^{2} \sin ^{2} \theta
$$

Similarly,

$$
e^{2 \frac{\pi}{a} x}\left(\cos ^{2} \frac{\pi}{a} y+\sin ^{2} \frac{\pi}{a} y\right)=r^{2}\left(\cos ^{2} \theta+\sin ^{2} \theta\right)
$$

This is

$$
\frac{e^{\frac{\pi}{a} x} \sin \frac{\pi}{a} y}{e^{\frac{\pi}{a} x} \cos \frac{\pi}{a} y}=\frac{r \sin \theta}{r \cos \theta}=\operatorname{Tan} \theta
$$

$\operatorname{Tan} \theta=\tan \frac{\pi}{a} y$,

thus

This means that

$$
\theta=\frac{\pi}{a} y
$$

2.3 Solution of the transformed problem

$$
w(r, \theta)=w\left(e^{\frac{\pi}{a} x}, e^{\frac{\pi}{a} y}\right)
$$

We now solve the transformed problem

$$
\begin{gathered}
\frac{\partial^{2} w}{\partial r^{2}}+\frac{1}{r} \frac{\partial w}{\partial r}+\frac{1}{r^{2}} \frac{\partial^{2} w}{\partial \theta^{2}}=0 \quad r \geq 0,0 \leq \theta \leq \pi \\
w(r, \pi)=T, \quad 0 \leq r \leq 1 \\
=0 \quad r>1 \\
w(r, 0)=2 \pi \quad 0 \leq r \leq 1 \\
=0 \quad r>1
\end{gathered}
$$

The asymptotic behavior of $w(r, 0) \approx r^{\circ}$ as $r \rightarrow 0$ and $w(r, \pi) \approx r^{-\frac{1}{2}}$ as $r \rightarrow \infty$

By use of the mellin integral transform defined by

Equation (2.5)-(2.7) are transformed into

$$
\bar{w}(s, \theta)=\int_{0}^{\infty} r^{s-1} w(r, \theta) d r \quad 0<\operatorname{Res}<\frac{1}{2}
$$

$$
\begin{gathered}
\frac{d^{2} \bar{w}}{d \theta^{2}}+s^{2} \bar{w}=0 \\
\bar{w}(s, \pi)=T / s \\
\bar{w}(s, 0)=2 T / s
\end{gathered}
$$

The solution of the ordinary differential equation (2.8) is considered as

$$
\bar{w}(s, \theta)=A \sin s \theta+B \operatorname{coss} \theta
$$

The constants A and B are determined from the boundary conditions, now from (2.11) and (2.10) we get

And

$$
\bar{w}(s, 0)=B=2 T / s
$$

That is

$$
\bar{w}(s, \pi)=A \sin \pi s+B \cos \pi s=T / s
$$

$$
\begin{aligned}
A \sin \pi s & =T / S-2 T / S \cos \pi s \\
A & =\frac{T}{S}\left(\frac{1-2 \cos \pi s}{\operatorname{Sin} \pi s}\right)
\end{aligned}
$$

Hence (2.11) becomes

$$
\bar{w}(s, \theta)=T\left\{\frac{1-2 \cos \pi s}{\sin \pi s}\right\} \frac{\sin s \theta}{s}+\frac{2 \pi}{s} \cos S \theta
$$

\section{4 solution of the original problem}

We next apply the inverse formula to get the potential sought for in the form 
Where $\bar{w}(s, 0)$ is given in $(2.12)$

$$
\bar{w}(r, \theta)=\frac{1}{2 \pi i} \int_{c-i \infty}^{c+i \infty} \bar{w}(s, \theta) r^{-s} d s, \quad 0<c<\frac{1}{2}
$$

The contour integral in (2.13) can be evaluated by method of Cauchy integral (or residue) theorem [3].

The poles of $\bar{w}(s, \theta)$ are all simple, these simple poles are located at $s= \pm n, n=1,2,3, \ldots$ and at $s=0$

At the poles $s=n, n=1,2,3,4, \ldots$, the residues are

Residue

Hence for $n=1,2,3,4, \ldots .(s=n)$

$$
\begin{aligned}
\bar{w}(r, \theta) r^{-s} & =\lim _{s \rightarrow n}(s-n) \bar{w}(s, \theta) r^{-s} \\
& =\lim _{s \rightarrow n}\left\{T\left(\frac{s-n}{\sin \pi s}\right)\left(\frac{1-2 \cos \pi s}{s}\right) \sin s \theta+\frac{2 T(s-n)}{s} \cos s \theta\right\} r^{-s} \\
& =\frac{T}{\pi} \lim _{s-n}\left\{\frac{1}{\cos \pi s}\left(\frac{1-2 \cos \pi s}{s}\right) \sin s \theta+0\right\} r^{-s} \\
& =\frac{T}{\pi}\left\{\frac{1}{\cos n \pi}\left(\frac{1-2 \cos n \pi}{s}\right) \sin n \theta+0\right\} r^{-n} \\
& =\frac{T}{\pi}(-1)^{n}\left(1-2(-1)^{n}\right) \frac{\sin n \theta}{n} r^{-n} \\
& =\frac{T}{\pi}\left((-1)^{n}-2\right) \frac{\sin n \theta}{n} r^{-n}
\end{aligned}
$$

$$
\bar{w}(r, \theta)=\frac{T}{\pi} \sum_{n=1}^{\infty}\left[\frac{(-1)^{n}-2}{n}\right] r^{-n} \sin n \theta
$$

At the poles $s=-n, n=1,2,3, \ldots$, the residues are

Hence for $n=1,2,3 \ldots . .(s=-n)$

$$
\begin{aligned}
\lim _{s \rightarrow n}(s+n) \bar{w}(s, \theta) r^{-s} & =T \lim _{s \rightarrow n} \frac{(s+n)}{\sin \pi s}\left(\frac{1-2 \cos \pi s}{s}\right) \sin s \theta r^{-s} \\
& =\frac{T}{\pi} \lim _{s \rightarrow n}\left\{\frac{1}{\cos \pi s}\left(\frac{1-2 \cos \pi s}{s}\right) \sin \theta \theta r^{-s}\right\} \\
& =\frac{T}{\pi}(-1)^{n}\left(\frac{1-2(-1)^{n}}{-n}\right) \sin (-n) \theta r^{n} \\
& =\frac{T}{\pi}(-1)^{n}\left(\frac{1-2(-1)^{n}}{n}\right) r^{n} \sin n \theta \\
& =\frac{T}{\pi}\left(\frac{(-1)^{n}-2}{n}\right) r^{n} \sin n \theta
\end{aligned}
$$

$$
\bar{w}_{2}(r, \theta)=\frac{T}{\pi} \sum_{n-1}^{\infty}\left[\frac{(-1)^{n}}{n}-2\right] r^{n} \sin n \theta
$$

Residue at $s=0$ is derived in the following way

Hence the solution-sought for is

$$
\begin{aligned}
\lim _{s \rightarrow 0}(s-0) \bar{w}(s, 0) r^{-s} & =\lim \left\{T \frac{s}{\sin \pi s}(1-2 \cos \pi s) \frac{\sin s \theta}{s}+\frac{2 T s}{s} \cos \theta\right\} \\
& =\lim \left\{\frac{T}{\pi} \frac{\pi s}{\sin \pi s}(1-2 \cos \pi s) \theta \frac{\sin \theta}{\theta s}+2 T \cos \theta\right\} \\
& =\frac{T}{\pi} \cdot 1 \cdot \theta \cdot 1+2 T \\
& =2 T-\frac{\theta T}{\pi}
\end{aligned}
$$

Thus solution is closed.

$$
\begin{gathered}
w(r, \theta)=2 T-\theta \frac{T}{\pi}+\frac{T}{\pi} \sum_{n=1}^{\infty}\left[\frac{(-1)^{n}-2}{n}\right] r^{n} \sin n \theta, \quad r<1 \\
=\frac{T}{\pi} \sum_{n=1}^{\infty}\left[\frac{(-1)^{n}-2}{n}\right] r^{-n} \sin n \theta, \quad r>1
\end{gathered}
$$

\subsection{VERIFICATION OF RESULT}

In the section, we as certain that (2.14) is indeed the solution of (2.5)-(2.7)

\section{5(a) SATISFACTION OF THE LAPLACE EQAUTION}


Since

$$
\begin{gathered}
w(r, \theta)=2 T-\theta \frac{T}{\pi}+\frac{T}{\pi} \sum_{n=1}^{\infty}\left[\frac{(-1)^{n}-2}{n}\right] r^{n} \sin n \theta, \quad r<1 \\
=\frac{T}{\pi} \sum_{n=1}^{\infty}\left[\frac{(-1)^{n}-2}{n}\right] r^{-n} \sin n \theta, \quad r>1
\end{gathered}
$$

It follows that:

1. For $r<1$

$$
\begin{aligned}
\frac{\partial W}{\partial r} & =\frac{T}{\pi} \sum_{n=1}^{\infty}\left[(-1)^{n}-2\right] r^{n-1} \sin n \theta \\
\frac{\partial^{2} W}{\partial r^{2}} & =\frac{T}{\pi} \sum_{n=1}^{\infty}(n-1)\left[(-1)^{n}-2\right] r^{n-2} \sin n \theta, \\
\frac{\partial W}{\partial \theta} & =-\frac{T}{\pi}+\frac{T}{\pi} \sum_{n=1}^{\infty}\left[(-1)^{n}-2\right] r^{n} \cos n \theta, \\
\frac{\partial^{2} W}{\partial \theta^{2}} & =-\frac{T}{\pi} \sum_{n=1}^{\infty} n\left[(-1)^{n}-2\right] r^{n} \sin n \theta,
\end{aligned}
$$

Therefore,

$$
\begin{aligned}
\frac{\partial^{2} W}{\partial r^{2}}+\frac{1}{r} \frac{\partial W}{\partial r}+\frac{1}{r^{2}} \frac{\partial^{2} W}{\partial \theta^{2}}= & \frac{T}{\pi} \sum_{n=1}^{\infty}(n-1)\left[(-1)^{n}-2\right] r^{n-2} \sin n \theta \\
& +\frac{T}{\pi} \sum_{n=1}^{\infty}\left[(-1)^{n}-2\right] r^{n-2} \sin n \theta \\
& -\frac{T}{\pi} \sum_{n=1}^{\infty}\left[(-1)^{n}-2\right] r^{n-1} \sin n \theta=\theta
\end{aligned}
$$

ii. for $r>1$

$$
\begin{aligned}
\frac{\partial W}{\partial r} & =-\frac{T}{\pi} \sum_{n=1}^{\infty}\left[(-1)^{n}-2\right] r^{-n-1} \sin n \theta \\
\frac{\partial^{2} W}{\partial r^{2}} & =\frac{T}{\pi} \sum_{n=1}^{\infty}(n-1)\left[(-1)^{n}-2\right] r^{-n-2} \sin n \theta \\
\frac{\partial W}{\partial \theta} & =\frac{T}{\pi} \sum_{n=1}^{\infty}\left[(-1)^{n}-2\right] r^{-n} \cos n \theta \\
\frac{\partial^{2} W}{\partial \theta^{2}} & =-\frac{T}{\pi} \sum_{n=1}^{\infty} n\left[(-1)^{n}-2\right] r^{-n} \sin n \theta
\end{aligned}
$$

Therefore

$$
\begin{aligned}
\frac{\partial^{2} W}{\partial r^{2}}+\frac{1}{r} \frac{\partial W}{\partial r}+\frac{1}{r^{2}} \frac{\partial^{2} W}{\partial \theta^{2}}= & \frac{T}{\pi} \sum_{n=1}^{\infty}(n+1)\left[(-1)^{n}-2\right] r^{-n-2} \sin n \theta \\
& -\sum_{n=1}^{\infty}\left[(-1)^{n}-2\right] r^{-n-2} \sin n \theta \\
& -\sum_{n=1}^{\infty} n\left[(-1)^{n}-2\right] r^{n-1} \sin n \theta=\theta
\end{aligned}
$$

Thus the Laplace equation (3.1.1) is satisfied for $r \geq 0$

\section{5(b) SATISFACTION OF BOUNDARY CONDITIONS}

i. $\quad$ For $r<1$

$$
\begin{aligned}
W(\mathrm{r}, \pi) & =2 T-\pi \frac{T}{\pi}+\frac{T}{\pi} \sum_{n=1}^{\infty}\left[\frac{(-1)^{n}-2}{n}\right] r^{n} \sin n \pi \\
& =2 T-T+0
\end{aligned}
$$


For $r>1$

$$
=T
$$

ii. For $r<1$

$$
W(\mathrm{r}, \pi)=\frac{T}{\pi} \sum_{n=1}^{\infty}\left[\frac{(-1)^{n}-2}{n}\right] r^{-n} \sin n \pi
$$

$$
\begin{aligned}
W(\mathrm{r}, 0) & =2 T-0 \frac{T}{\pi}+\frac{T}{\pi} \sum_{n=1}^{\infty}\left[\frac{(-1)^{n}-2}{n}\right] r^{-n} \sin 0 n \\
& =2 T
\end{aligned}
$$

For $r>1$

$$
\begin{aligned}
W(\mathrm{r}, 0) & =\frac{T}{\pi} \sum_{n=1}^{\infty}\left[\frac{(-1)^{n}-2}{n}\right] r^{-n} \sin 0 n \\
& =0
\end{aligned}
$$

Therefore the boundary conditions are satisfied for both $\mathrm{r}<1$ and $\mathrm{r}>1$

\subsection{CONCLUSION}

From

As

$$
w(r, \theta)=w\left(e^{\frac{\pi}{a} x}, e^{\frac{\pi}{a} y}\right)=W(u, v)
$$

Together with the harmonicity condition

$$
\emptyset(x, y)=W(u, v)
$$

We get the original potential with

$$
r=e^{\frac{\pi}{a} x} \text { and } \theta=\frac{\pi}{a} y
$$

$$
\begin{array}{rlr}
\emptyset(x, y) & =2 T-\frac{\pi}{a} y \frac{T}{\pi}+\frac{T}{\pi} \sum_{n=1}^{\infty}\left[\frac{(-1)^{n}-2}{n}\right] e^{n \frac{\pi}{a} x} \sin n \frac{\pi}{a} y, & e^{\frac{\pi}{a} x}<1 \\
& =\frac{T}{\pi} \sum_{n=1}^{\infty}\left[\frac{(-1)^{n}-2}{n}\right] e^{-n \frac{\pi}{a} x} \operatorname{sinn} \frac{\pi}{a} y, & e^{\frac{\pi}{a} x}>1
\end{array}
$$

\section{References}

[1] I.N Sneddon : The use of integral transforms, McGraw-Hill,new York,1972

[2] G.N.Emenogu and J.N.Nnadi: Analysis of elastic wedge under out-of-plane stress volume 66,number 1, pp117-125(2012)

[3] Z. Szmydt and B.Ziemian, The Mellin transform and fuchsia type partial differential equations, mathematics and its applications (East Europe Series)56,kluwer Academic publishers Group, Dordrech, 1992

[4] J.N.Nnadi: Anti plane shear analysis for a Non-homogeneous semi-infinite layer. Journal of the Nigerian Association of mathematical physis,volume7,p215(2003) 